\title{
Order Statistics Properties of the Two Parameter Lomax Distribution
}

Javid Gani Dar

Department of Computer Science Engineering

Unit: Mathematics and Statistics, School of Technology

Islamic University of Science and Technology

Awantipora, Pulwama Jammu and Kashmir India

javinfo.stat@yahoo.co.in

Abdullah Al-Hossain

Department of Mathematics, Jazan University

Kingdom of Saudi Arabia

aalhossain@jazanu.edu.sa

\begin{abstract}
In this paper we study the distribution of order statistics of the two parametric Lomax distribution. We consider the single and product moment of order statistics from Lomax distribution. Also, we establish some recurrence relation for single moments of order statistics. The exact analytical expressions of entropy, residual entropy and past residual entropy for order statistics of Lomax distribution is derived.
\end{abstract}

AMS Subject Classification: 62G30; 94A17; 60E05.

Key words: Lomax distribution, Order statistics, Moments, Single and product moment of order statistics, Entropy, Residual entropy.

\section{Introduction}

Order statistics have been used in wide range of problems, including robust statistical estimation and detection of outliers, characterization of probability distribution, goodness of fit-tests, quality control, analysis of censored sample, etc; see (Arnold et al., 1992; Beirlant et al., 1997; Tahir et al., 2015). The use of recurrence relations for the moments of order statistics is quite well known in statistical literature (see for example Arnold et al., (1992), Malik et al. (1998). For improved form of these results, Samuel and Thomes (2000), Arnold et al. (1992) have reviewed many recurrence relations and identities for the moments of order statistics arising from several specific continuous distributions such as normal, Cauchy, logistic, gamma and exponential. The Lomax distribution is often used in business, economics and actuarial modeling. It was used by Lomax (1954) to fit date in business failure. For various properties and applications of Lomax distribution one should refer to (Ghitany et al., 2007; Giles et al., 2013).

We say that a random variable $X$ with range of values $(0, \infty)$ has the two parametric Lomax distribution (now onwards Lomax distribution) if its pdf is given by

$$
f(x)=\frac{\alpha}{\lambda}\left(1+\frac{x}{\lambda}\right)^{-(\alpha+1)}, x>0, \alpha, \lambda>0
$$

The cumulative distribution function (cdf) and survival function (sf) associated with (1.1) is given by

$$
F(x)=1-\left(1+\frac{x}{\lambda}\right)^{-\alpha}
$$


respectively.

$$
\bar{F}(x)=\left(1+\frac{x}{\lambda}\right)^{-\alpha}
$$

The following functional relationship exists between p.d.f and c.d.f of Lomax distribution:

$$
f(x)=\alpha(\lambda+x)^{-1}-\alpha(\lambda+x)^{-1} F(x) .
$$

The idea of entropy of a random variable $X$ was developed by Claude Shannon (1948) for the first time in information theory. The differential entropy of a continuous random variable $X$ with density function $f_{X}(x)$ is defined as

$$
H(X)=-\int_{0}^{\infty} f_{X}(x) \log f_{X}(x) d x
$$

Analytical expression for univerate distribution are discussed in references such as Laz and Rathie (1978), Nadarajah and Zagrafos (2003), etc. Also the information properties of order statistics have been studied by Wong and Chen (1990), Park (1995), Ebrahimi et al. (2004), etc.

In case on has information about the current age of the component, which can take into account for measuring its uncertainty, then the measure given in (1.5) is not suitable. A more realistic approach which make the use of the current age into account is described by Ebrahimi (1996) and is defined as

$$
H(X ; t)=-\int_{t}^{\infty} \frac{f(x)}{\bar{F}(t)} \log \frac{f(x)}{\bar{F}(t)} d x
$$

where $\bar{F}(t)$ is the survival function. For $t=0,(1.6)$ reduces to (1.5).

In many realistic situation uncertainty is not necessarily related to future but can also refer to past. Based on this idea, Crescenzo and Longobardi (2004) develop the concept of past entropy over $(0, t)$. If $X$ denote the lifetime of a component or of living organism, then the past entropy of $X$ is defined as

$$
H^{0}(X ; t)=-\int_{0}^{t} \frac{f(x)}{F(t)} \log \frac{f(x)}{F(t)} d x,
$$

where $F(t)$ is the cumulative distribution function. For $t=\infty,(1.7)$ reduces to (1.5).

\section{Distribution of Order Statistics}

Let $X_{1}, X_{2}, \ldots, X_{n}$ be a random sample of size $n$ from the Lomax distribution and let $X_{1: n} \leq X_{2: n} \leq \cdots \leq X_{n: n}$ denotes the corresponding order statistics. Then the pdf of $X_{r: n}, 1 \leq r \leq n$, is given by [see David and Nagaraja (1981) and Arnold et al. (1992)

$$
f_{r: n}(x)=C_{r: n}\left\{[F(x)]^{r-1}[1-F(x)]^{n-r} f(x)\right\}, 0<x<\infty,
$$

where $C_{r: n}=\frac{n !}{(r-1) !(n-r) !}$. 
The probability density function of smallest $(r=1)$ and largest $(r=n)$ order statistics can be easily obtained from (2.1) and is given by $f_{(1, n)}(x)=n[1-F(x)]^{n-1} f(x)$ and $f_{(n, n)}(x)=n[F(x)]^{n-1} f(x)$ respectively.

Also, the cumulative distribution function of the largest and smallest order statistics is given by $F_{(n, n)}(x)=[F(x)]^{n}$ and $F_{(1, n)}(x)=1-[1-F(x)]^{n}$ respectively.

Using (1.1), (1.2) and taking $r=1$ in (2.1), yields the pdf of the minimum order statistics for the Lomax distribution

$$
f_{1: n}(x)=\frac{n \alpha}{\lambda}\left(1+\frac{x}{\lambda}\right)^{-n \alpha-1}
$$

Similarly using (1.1), (1.2) and taking $r=n$ in (2.1), yields the pdf of the largest order statistics for the Lomax distribution

$$
f_{n: n}(x)=\frac{n \alpha}{\lambda}\left[1-\left(1+\frac{x}{\lambda}\right)^{-\alpha}\right]^{n-1}\left(1+\frac{x}{\lambda}\right)^{-(\alpha+1)}
$$

The joint pdf of $X_{r: n}$ and $X_{s: n}$ for $1 \leq r<s \leq n$ is given by [see Arnold et al. [4]

$$
\begin{aligned}
& f_{r, s: n}(x)=C_{r, s: n}\left\{[F(x)]^{r-1}[F(y)-F(x)]^{s-r-1}[1-F(y)]^{n-s} f(x) f(y)\right\} \\
& \text { for }-\infty<x<y<\infty \text { and } C_{r, s: n}=\frac{n !}{(r-1) !(s-r-1) !(n-s) !} .
\end{aligned}
$$

Following two theorems gives the distribution of the order statistics from the Lomax distribution.

Theorem 2.1: Let $F(x)$ and $f(x)$ be the cdf and pdf of the Lomax distribution. Then the density function of the $r^{\text {th }}$ order statistics say $f_{r: n}(x)$ is given by

$$
f_{r: n}(x)=\alpha C_{r: n} \sum_{i=0}^{n-r} \sum_{j=0}^{r+i-1}\left(\begin{array}{c}
n-r \\
i
\end{array}\right)\left(\begin{array}{c}
r+i-1 \\
j
\end{array}\right)(-1)^{i+j}\left[\lambda^{\alpha(j+1)}(\lambda+x)^{-\alpha(j+1)-1}\right]
$$

Proof: See Appendix A

Theorem 2.2: Let $X_{r: n}$ and $X_{s: n}$ for $1 \leq r<s \leq n$ be the $r^{\text {th }}$ and $s^{\text {th }}$ order statistics from the Lomax distribution. Then the joint pdf of $X_{r: n}$ and $X_{s: n}$ is given by

$$
\begin{gathered}
f_{r ; s: n}(x)=\alpha^{2} C_{r: n} \sum_{i=0}^{s-r-1} \sum_{j=0}^{n-s}\left(\begin{array}{c}
s-r-1 \\
i
\end{array}\right)\left(\begin{array}{c}
n-s \\
j
\end{array}\right)(-1)^{i+j} \times \\
\frac{1}{\lambda^{-\alpha(s+j)}}\left[\lambda^{-\alpha}-(\lambda+y)^{-\alpha}\right]^{s-r-1-i+j}\left[\lambda^{-\alpha}-(\lambda+x)^{-\alpha}\right]^{r+i-1}(\lambda+x)^{-(\alpha+1)}(\lambda \\
+y)^{-(\alpha+1)} .
\end{gathered}
$$

Proof: See Appendix A 


\section{Single and Product Moments}

In this section, we derive explicit expressions for both of the single and product moments of order statistics from the Lomax distribution.

Theorem 3.1: Let $X_{1}, X_{2}, \ldots, X_{n}$ be a random sample of size $n$ from the Lomax distribution and let $X_{1: n} \leq X_{2: n} \leq \cdots \leq X_{n: n}$ denote the corresponding order statistics. Then the $k^{t h}$ moments of the $r t h$ order statistics for $k=1,2, \ldots$. denoted by $\mu_{r: n}^{(k)}$ is given by

$\mu_{r: n}^{(k)}=\alpha C_{r: n} \sum_{l=0}^{n-r} \sum_{i=0}^{k} \sum_{j=0}^{l+r-1}\left(\begin{array}{c}n-r \\ l\end{array}\right)\left(\begin{array}{l}k \\ i\end{array}\right)\left(\begin{array}{c}l+r-1 \\ j\end{array}\right)(-1)^{l+i+j}\left(\frac{1}{i+\alpha j+\alpha-k}\right)$,

where the value of $\alpha$ is chosen in such a way that $\left(\frac{i-k}{\alpha}+j\right) \neq-1$.

Proof: See Appendix B

Now we derive recurrence the relation for single moments.

Theorem 3.2: Let $X_{1}, X_{2}, \ldots, X_{n}$ be a random sample of size $n$ from the Lomax distribution and let $X_{1: n} \leq X_{2: n} \leq \cdots \leq X_{n: n}$ denote the corresponding order statistics. Then for $1 \leq r \leq n$, we have the following moment relation:

$$
\begin{aligned}
\mu_{r: n}^{(k)}= & \sum_{i=0}^{k-1} \sum_{j=0}^{k-i}\left(\begin{array}{c}
k \\
i
\end{array}\right)\left(\begin{array}{c}
k-i \\
j
\end{array}\right)(-1)^{i} \lambda^{k-i-j}\left\{\alpha n\left(\mu_{r: n-1}^{(j)}-\mu_{r+1: n-1}^{(j)}\right)-\alpha r\left(\mu_{r+1: n}^{(j)}-\mu_{r: n}^{(j)}\right)\right\} \\
+ & C_{r: n}\left\{\alpha\left(A_{1}-A_{2}-A_{3}+A_{4}\right)+(r-1) \sum_{l=0}^{r-2}\left(\begin{array}{c}
r-2 \\
l
\end{array}\right)(-1)^{l} \frac{1}{(n+l-r+1)^{2}}\right. \\
& -(n-r) \sum_{s=0}^{r-1}\left(\begin{array}{c}
r-1 \\
s
\end{array}\right)(-1)^{s} \frac{1}{(n+s-r)^{2}} \\
& -r \sum_{l=0}^{r-1}\left(\begin{array}{c}
r-1 \\
l
\end{array}\right)(-1)^{l} \frac{1}{(n+l-r+1)^{2}} \\
& \left.-(n-r) \sum_{s=0}^{r}\left(\begin{array}{l}
r \\
s
\end{array}\right)(-1)^{s} \frac{1}{(n+s-r)^{2}}\right\}
\end{aligned}
$$

where $A_{1}=(r-1) \log \lambda B(n-r ; r-1), A_{2}=(n-r) \log \lambda B(n-r ; r)$

$A_{3}=r \log \lambda B(n-r ; r), A_{4}=(n-r) \log \lambda B(n-r ; r)$ and $B(m ; n)$ is the beta function.

Proof: See Appendix B 
Theorem 3.3: For $1 \leq r \leq s \leq n, n \in N$, we have

$\mu_{r ; s: n}^{\left(k_{1}, k_{2}\right)}=$ $\alpha^{2} C_{r: s ; n} \sum_{i=0}^{n-s} \sum_{j=0}^{s-r-1} \sum_{l=0}^{k_{2}} \sum_{m=0}^{s-r-j+i-1} \sum_{p=0}^{k_{1}} \sum_{q=0}^{j+r-1}\left(\begin{array}{c}n-s \\ i\end{array}\right)\left(\begin{array}{c}s-r-1 \\ j\end{array}\right)\left(\begin{array}{c}k_{2} \\ l\end{array}\right)\left(\begin{array}{c}s-r-j+i-1 \\ m\end{array}\right)\left(\begin{array}{c}k_{1} \\ p\end{array}\right)\left(\begin{array}{c}j+r-1 \\ q\end{array}\right) \times$ $\lambda^{k_{1}+k_{2}}(-1)^{i+j+l+m+p+q}\left\{\frac{1}{\left(l-k_{2}+\alpha m+\alpha\right)\left(p-k_{1}+l-k_{2}+\alpha q+\alpha m+2 \alpha\right)}\right\}$

Proof: See Appendix B

\section{Applications}

For $k=1, \alpha \geq 1$ we obtain the mean of the $r$ th order statistic as:

$$
\begin{aligned}
\mu_{r: n} & =E\left(X_{r: n}\right)=\int_{0}^{\infty} x f_{r: n}(x) d x \\
& =C_{r, n} \int_{0}^{\infty} x[F(x)]^{r-1}[1-F(x)]^{n-r} f(x) d x \\
& =\frac{\alpha C_{r, n}}{\lambda} \int_{0}^{\infty} x\left[1-\left(1+\frac{x}{\lambda}\right)^{-\alpha}\right]^{r-1}\left[\left(1+\frac{x}{\lambda}\right)^{-\alpha}\right]^{n-r}\left(1+\frac{x}{\lambda}\right)^{-\alpha-1} d x \\
& =\alpha \lambda C_{r, n} \sum_{i=0}^{r-1} \frac{\left(\begin{array}{c}
r-1 \\
i
\end{array}\right)(-1)^{i}}{\alpha(n-r+i+1)-1}-\lambda .
\end{aligned}
$$

Now for $k=2, \alpha \geq 2$, one can get the second order moment of the $r$ th order statistic as

$\mu_{r: n}^{(2)}=E\left(X_{r: n}^{2}\right)=\int_{0}^{\infty} x^{2} f_{r: n}(x) d x$

$=\alpha \lambda^{2} C_{r, n} \sum_{i=0}^{r-1} \frac{\left(\begin{array}{c}r-1 \\ i\end{array}\right)(-1)^{i}}{\alpha(n-r+i+1)-2}-2 \alpha \lambda^{2} C_{r, n} \sum_{i=0}^{r-1} \frac{\left(\begin{array}{c}r-1 \\ i\end{array}\right)(-1)^{i}}{\alpha(n-r+i+1)-1}+\lambda^{2}$.

Therefore the variance of the $r$ th order statistic can be obtained easily by using the relation

$$
\begin{aligned}
V\left(X_{r: n}\right) & =\mu_{r: n}^{(2)}-\left(\mu_{r: n}\right)^{2} \\
& =\alpha \lambda^{2} C_{r, n} \sum_{i=0}^{r-1} \frac{\left(\begin{array}{c}
r-1 \\
i
\end{array}\right)(-1)^{i}}{\alpha(n-r+i+1)-2}-\left(\alpha \lambda C_{r, n} \sum_{i=0}^{r-1} \frac{\left(\begin{array}{c}
r-1 \\
i
\end{array}\right)(-1)^{i}}{\alpha(n-r+i+1)-1}\right)^{2} .
\end{aligned}
$$

The mean, variance and other statistical measure of the extreme order statistics are always of great interest. Taking $r=1$, one can obtain the mean of smallest order statistics:

$$
\mu_{1: n}=\frac{n \lambda \alpha}{n \alpha-1}-\lambda
$$

Also, second order moment of the smallest order statistic can be obtained as:

$$
\mu_{1: n}^{(2)}=\frac{n \alpha \lambda^{2}}{n \alpha-2}-\frac{2 n \alpha \lambda^{2}}{n \alpha-1}+\lambda^{2} \text {. }
$$


Therefore

$$
\begin{aligned}
& V\left(X_{1: n}\right)=\mu_{1: n}^{(2)}-\left(\mu_{1: n}\right)^{2} \\
& =\frac{n \alpha \lambda^{2}}{n \alpha-2}-\left(\frac{n \lambda \alpha}{n \alpha-1}\right)^{2} .
\end{aligned}
$$

Similarly mean, second order moment and hence variance of the largest order statistics $(r=n)$ can be easily obtained as

$$
\begin{aligned}
& \mu_{n: n}=\lambda \alpha n \sum_{i=0}^{n-1} \frac{\left(\begin{array}{c}
n-1 \\
i
\end{array}\right)(-1)^{i}}{\alpha(i+1)-1}-\lambda . \\
& \mu_{n: n}^{(2)}=n \alpha \lambda^{2} \sum_{i=0}^{n-1} \frac{\left(\begin{array}{c}
n-1 \\
i
\end{array}\right)(-1)^{i}}{\alpha(i+1)-2}-2 n \alpha \lambda^{2} \sum_{i=0}^{n-1} \frac{\left(\begin{array}{c}
n-1 \\
i
\end{array}\right)(-1)^{i}}{\alpha(i+1)-1}+\lambda^{2} . \\
& V\left(X_{n: n}\right)=n \alpha \lambda^{2} \sum_{i=0}^{n-1} \frac{\left(\begin{array}{c}
n-1 \\
i
\end{array}\right)(-1)^{i}}{\alpha(i+1)-2}-\left(\lambda \alpha n \sum_{i=0}^{n-1} \frac{\left(\begin{array}{c}
n-1 \\
i
\end{array}\right)(-1)^{i}}{\alpha(i+1)-1}\right)^{2} .
\end{aligned}
$$

\section{Entropy for the Lomax Distribution Based on Order Statistics}

Let $X_{1}, X_{2}, \ldots, X_{n}$ be a random sample of size $n$ from a distribution $F_{X}(x)$ with density function $f(x)$ and let $Y_{1}<Y_{2}<\cdots<Y_{n}$ denote the corresponding order statistics. Then the pdf of $Y_{r}, 1 \leq r \leq n$, is given by

$$
f_{Y_{r}}(y)=C_{r: n}\left\{\left[F_{X}(y)\right]^{r-1}\left[1-F_{X}(y)\right]^{n-r} f(x)\right\}, 0<x<\infty
$$

where $\quad C_{r: n}=\frac{1}{B(r, n-r+1)}$ and $B(r, n-r+1)=\frac{\Gamma(\mathrm{r}) \Gamma(\mathrm{n}-\mathrm{r}+1)}{\Gamma(\mathrm{n}+1)}=\frac{(\mathrm{r}-1) !(\mathrm{n}-\mathrm{r}) !}{\mathrm{n} !} \quad$ is the beta function.

Let $U$ is the uniform distribution defined over the unit interval. The order statistics of a sample from uniform distribution $U_{1}, U_{2}, \ldots U_{n}$ are denoted by $W_{1}<W_{2}<\cdots W_{n}$ and $W_{r}, r=1,2, \ldots, n$ has beta distribution with density function

$$
g_{r}(w)=C_{r: n}\left\{[w]^{r-1}[1-w]^{n-r}\right\}, 0<w<1 .
$$

Now we derive the exact form of entropy, residual entropy and past residual entropy for the Lomax distribution based on order statistics:

\section{(i) Entropy (See Shannon [22 ])}

Using the transformation $W_{r}=F_{X}\left(Y_{r}\right)$, the entropies of order statistics can be computed by

$$
H\left(Y_{r}\right)=H_{n}\left(W_{r}\right)-E_{g_{r}}\left[\log f_{X}\left(F_{X}^{-1}\left(W_{r}\right)\right)\right]
$$


Where $f_{X}$ is probability density function of the random variable $X$ and $H_{n}\left(W_{r}\right)$ denote the entropy of the beta distribution and is given by

$$
\begin{gathered}
H_{n}\left(W_{r}\right)=\log B(r, n-r+1)-(r-1)[\psi(r)-\psi(n+1)] \\
-(n-r)[\psi(n-r+1)-\psi(n+1)],
\end{gathered}
$$

where $\psi$ is the digamma function and is defined by $\psi(\theta)=\frac{d}{d \theta} \log \Gamma(\theta)$.

Remark 4.1: For $r=1$ (i.e smallest order statistics) and $r=n$ (i.e largest order statistics), it can be easily shown that

$$
H_{n}\left(W_{1}\right)=H_{n}\left(W_{n}\right)=1-\log n-\frac{1}{n}
$$

Remark 4.2: It should be noted that $\psi(n+1)-\psi(n)=\frac{1}{n}$.

Theorem 4.1: Let $X_{1}, X_{2}, \ldots, X_{n}$ be a random sample of size $n$ from Lomax distribution with distribution function given in (1.2) and let $Y_{1}<Y_{2}<\cdots<Y_{n}$ denote the corresponding order statistics. Then the entropy of the $r^{\text {th }}$ order statistics for the Lomax distribution is given by

$$
\begin{aligned}
H\left(Y_{r}\right)= & \log B(r, n-r+1)-(r-1)[\psi(r)-\psi(n+1)] \\
& -(n-r)[\psi(n-r+1)-\psi(n+1)]-\log \frac{\alpha}{\lambda}+\frac{\alpha+1}{\alpha}[\psi(n-r+1)-\psi(n+1)]
\end{aligned}
$$

Proof: See Appendix C.

Corollary 4.1: For $r=1$ (i.e smallest order statistics):

$$
H\left(Y_{1}\right)=1-\log \frac{n \alpha}{\lambda}+\frac{1}{n \lambda \alpha} \text {. }
$$

Corollary 4.2: For $r=n$ (i.e largest order statistics):

$$
\begin{aligned}
H\left(Y_{n}\right) & =1-\frac{1}{n}-\log \frac{n \alpha}{\lambda}+\frac{\alpha+1}{\alpha}(\psi(n+1)-\psi(1)) \\
& =1-\frac{1}{n}-\log \frac{n \alpha}{\lambda}+\frac{\alpha+1}{\alpha}(\psi(n+1)+\gamma),
\end{aligned}
$$

where $-\psi(1)=\gamma=0.5772$ is the Eulers constant.

\section{(ii) Residual Entropy}

Analogous to (1.6), the residual entropy of order statistics $X_{r, n}$ is given by

$$
H\left(X_{r, n} ; t\right)=-\int_{t}^{\infty} \frac{f_{r, n}(x)}{\bar{F}_{r, n}(t)} \log \frac{f_{r, n}(x)}{\bar{F}_{r, n}(t)} d x .
$$

Clearly the residual entropy of first order statistics is obtained by substituting $r=1$ and using the probability integral transformation $U=F_{X}(x)$ in (4.4), we have

$$
H\left(X_{1, n} ; t\right)=\frac{n-1}{n}-\log n+\log \bar{F}(t)-\frac{n}{\bar{F}^{n}(t)} \int_{F(t)}^{1}(1-u)^{n-1} \log \left[f\left(F^{-1}(u)\right)\right] d u \text {. }
$$


The residual entropy of the first order statistics for Lomax distribution can be easily obtained by using (1.1), (1.2), (1.3) and $f\left(F^{-1}(u)\right)=\frac{\alpha}{\lambda}(1-u)^{\frac{\alpha+1}{\alpha}}$ in (4.5):

$$
H\left(X_{1, n} ; t\right)=\left(1+\frac{1}{n \alpha}\right)-\log \frac{n \alpha}{\alpha}+\log \left(1+\frac{t}{\alpha}\right) .
$$

The case for $r=n$ follows on similar lines.

\section{(iii) Past Residual Entropy}

Analogous to (1.7), the past residual entropy of the $r^{\text {th }}$ order statistics is defined as

$$
H^{0}\left(X_{r, n} ; t\right)=-\int_{0}^{t} \frac{f_{r, n}(x)}{F_{r, n}(t)} \log \frac{f_{r, n}(x)}{F_{r, n}(t)} d x
$$

The past residual entropy of $n^{\text {th }}$ order statistics is obtained by substituting $r=n$ and using the probability integral transformation $U=F_{X}(x)$ in (4.6), we have

$H^{0}\left(X_{n, n} ; t\right)=\frac{n-1}{n}-\log n+\log F(t)-\frac{n}{F^{n}(t)} \int_{0}^{F(t)}(u)^{n-1} \log \left[f\left(F^{-1}(u)\right)\right] d u$.

The past residual entropy of the $n^{\text {th }}$ order statistics for Lomax distribution can be easily obtained by using $(1.1),(1.2),(1.3)$ and $f\left(F^{-1}(u)\right)=\frac{\alpha}{\lambda}(1-u)^{\frac{\alpha+1}{\alpha}}$ in (4.7):

$$
\begin{aligned}
H^{0}\left(X_{n, n} ; t\right)= & \frac{n-1}{n}+\log F(t)-\log n-\log \frac{\alpha}{\lambda}-\frac{\alpha+1}{\alpha} \log \bar{F}(t) \\
& -\frac{\alpha+1}{\alpha F^{n}(t)}\left[\sum_{i=1}^{n}\left(\begin{array}{l}
n \\
i
\end{array}\right)(-1)^{i} \frac{1}{i}\left\{\left(1-F^{i}(t)\right)-\log \bar{F}(t)\right\}\right],
\end{aligned}
$$

where $F(t)$ and $\bar{F}(t)$ are the cumulative distribution function and survival function for Lomax distribution given in (1.3) and (1.4) respectively.

The case for $r=1$ follows on similar lines.

\section{Conclusion}

In this paper we study the distribution from the order statistics of Lomax distribution. Also we consider the single and product moment of order statistics from Lomax distribution. We establish recurrence relation for single moments of order statistics. Also, we have derived the entropy, residual and past residual entropies for order statistics of the Lomax distribution.

\section{References}

1. Abdul-Moniem, I. B. and Abdel-Hameed, H. F. (2012). On exponentiated Lomax distribution. International J. Math Arch, (3) 2144-2150.

2. Al-Awadhi, S. A. and Ghitany, M. E. (2001). Statistical properties of PoissonLomax distribution and its application to repeated accidents data. Journal of Applied Statistical Science, (10) 365-372. 
3. Ali, A. A. and Khan, A. H. (1998). Recurrence relations for the expected values of the certain function of two order statistics. Metron, LVI, 107-119.

4. Arnold, B. C., Blakrishan, N. and Nagraja, N. H. (1992). A first course in order Statistics. John Wiley and Sons, New York.

5. Bandere, A .Z. and Hanaa, S. (2014). The Poisson-Lomax distribution. Colombian Journal of Statistics, (37) 223-243.

6. Behboodian, j. and Tahmasebi, S. (2008). Some properties of entropy for the exponentiated pareto distribution (EPD) based on order statistics. Journal of Mathematical Extension, (3) 43-53.

7. Beirlant, J. et.,al (1997). Non parametric entropy estimation: And overview, Int. J. Math. Statist. Sci., (6) 17-39.

8. Crescenzo, A. D and Longobardi, M. (2004). Entropy based measure of uncertainty in past lifetime distribution. Journal of Applied probability, (39) 434-440.

9. David, H. A. (1981). Order Statistics. Second Edition, John Willey and Sons New York.

10. Ebrahimi, N (1996). How to measure uncertainty in the residual lifetime distribution. Sankhya Ser. A, (58) 48-56.

11. Ebrahimi, N. et.,al (2004). Information properties of order statistics and spacing. IEEE Trans. Information Theory: (50), 177-183.

12. Ghitany, M. E.., Al-Awadhi, F. A. and Alkhalfan, L. A. (2007). Marshall-Olkin extended Lomax distribution and its application to censored data. Commun. in Statistics- Theory and Method, (36 ) 1855-1866.

13. Giles D. E. et., al (2013). On the Bias of the Maximum Likelihood Estimator for the Two-Parameter Lomax Distribution. Commun in Statistics - Theory and Methods, (42) 1934-1950.

14. Khan, A. H. Yaqub, M. and Parvez, S. (1983). Recurrence relations between moments of order statistics. Naval. Res. Logist. Quart., (30) 419-441.

15. Laz, A.C. and Rathie, P.N. (1978). On the entropy of continuous probability distribution. IEEE transactions of Information Theory IT, (24) 120-122.

16. Lomax, K.S. (1954). Business failure: another example of the analysis of failure data. J. Amer. Statist. Assoc: (49) 847-852.

17. Mailk H. J., Balakrishnan, N. and Ahmad, S. E. (1998). Recurrence relations and identities for moments of order statistics 1: Arbitrary continuous distributions. Commun. Statist.-Theo.Meth., (17) 2623-2655.

18. Nadarajah, S. (2005b). Sums, products and ratios for the biverate Lomax distribution. Comput.Statist. Data Anal: (49)109-129.

19. Nadarajah, S. and Zagrafos, K (2003). Formulas for Renyi information and related measures for univaraite distributions. Information Sciences, (155) 119-138. 
20. Park, S. (1995). The entropy of consecutive order statistics. IEEE Trans. Inform. Theory, (41) 2003-2007.

21. Samuel, P. and Thomas, P. Y. (2000). An improved form of a recurrence relation on the product moment of order statistics. Commun. Statist.-Theo.Meth., (29) 1559-1564.

22. Shannon, C. E. (1948). A mathematical theory of communication. Bell System Technical Journal. (27), 379-423 and 623-656.

23. Tahir, M. H., et.,al (2015). The Weibull-Lomax distribution: properties and applications. Hacettepe Journal of Mathematics and Statistics, (44), 455 - 474.

24. Wong, K. M. and Chen, S. (1990). The entropy of ordered sequences and order statistics. IEEE Trans. Information Theory: (36), 276-284. 


\section{APPENDIX}

\section{Appendix A:}

\section{Proof of Theorem 2.1:}

Equation (2.1) can be written as

$$
f_{r: n}(x)=C_{r: n} \sum_{i=0}^{n-r}\left(\begin{array}{c}
n-r \\
i
\end{array}\right)(-1)^{i}[F(x)]^{r+i-1} f(x)
$$

The proof follows by substituting (1.1) and (1.2) into (A.1) and expanding the term $\left(\lambda^{-\alpha}-(\lambda+x)^{-\alpha}\right)^{r+i-1}$ using the binomial expansion.

\section{Proof of Theorem 2.2:}

Equation (2.2) can be written as

$$
\begin{aligned}
f_{r ; s: n}(x)= & C_{r: n} \sum_{i=0}^{s-r-1} \sum_{j=0}^{n-s}\left(\begin{array}{c}
s-r-1 \\
i
\end{array}\right)\left(\begin{array}{c}
n-s \\
j
\end{array}\right)(-1)^{i+j}[F(y)]^{s-r-1-i+j} \\
& \times[F(x)]^{r+i-1} f(x) f(y) .
\end{aligned}
$$

The proof follows by substituting (1.1) and (1.2) into (A.2).

\section{Appendix B:}

\section{Proof of Theorem 3.1:}

We know that

$$
\begin{aligned}
\mu_{r: n}^{(k)}= & E\left(X_{r: n}^{k}\right)=\int_{0}^{\infty} x^{k} f_{r: n}(x) d x \\
= & C_{r: n} \int_{0}^{\infty} x^{k}[F(x)]^{r-1}[1-F(x)]^{n-r} f(x) d x \\
= & C_{r: n} \sum_{l=0}^{n-r}\left(\begin{array}{c}
n-r \\
l
\end{array}\right)(-1)^{l} \int_{0}^{\infty} x^{k}[F(x)]^{l+r-1} f(x) d x \\
= & C_{r: n} \sum_{l=0}^{n-r}\left(\begin{array}{c}
n-r \\
l
\end{array}\right)(-1)^{l} \int_{0}^{\infty} x^{k}\left[1-\left(1+\frac{x}{\lambda}\right)^{-\alpha}\right]^{l+r-1} \\
& \times \frac{\alpha}{\lambda}\left(1+\frac{x}{\lambda}\right)^{-(\alpha+1)} d x
\end{aligned}
$$

Put $\left(1+\frac{x}{\lambda}\right)^{-\alpha}=z \Rightarrow \frac{\alpha}{\lambda}\left(1+\frac{x}{\lambda}\right)^{-(\alpha+1)} d x=-d z$

Therefore (B.1) becomes

$$
\mu_{r: n}^{(k)}=C_{r: n} \sum_{l=0}^{n-r} \sum_{i=0}^{k} \sum_{j=0}^{l+r-1}\left(\begin{array}{c}
n-r \\
l
\end{array}\right)\left(\begin{array}{c}
k \\
i
\end{array}\right)\left(\begin{array}{c}
l+r-1 \\
j
\end{array}\right)(-1)^{l+i+j} \lambda^{k} \int_{0}^{1} \mathrm{Z}^{\left(\frac{i-k+\alpha j}{\alpha}\right)} \mathrm{dz} .
$$


Or

$\mu_{r: n}^{(k)}=\alpha C_{r: n} \sum_{l=0}^{n-r} \sum_{i=0}^{k} \sum_{j=0}^{l+r-1}\left(\begin{array}{c}n-r \\ l\end{array}\right)\left(\begin{array}{l}k \\ i\end{array}\right)\left(\begin{array}{c}l+r-1 \\ j\end{array}\right)(-1)^{l+i+j}\left(\frac{1}{i+\alpha j+\alpha-k}\right)$.

Hence the theorem is proved

\section{Proof of Theorem 3.2:}

We know that

$$
\begin{aligned}
\mu_{r: n}^{(k)} & =\int_{0}^{\infty} x^{k} f_{r: n}(x) d x \\
& =C_{r: n} \int_{0}^{\infty} x^{k}[F(x)]^{r-1}[1-F(x)]^{n-r} f(x) d x
\end{aligned}
$$

Using (1.4), we get

$$
\begin{aligned}
\mu_{r: n}^{(k)}=\alpha C_{r: n} & \left\{\int_{0}^{\infty} x^{k}(\lambda+x)^{-1}[F(x)]^{r-1}[1-F(x)]^{n-r} d x\right. \\
& \left.-\int_{0}^{\infty} x^{k}(\lambda+x)^{-1}[F(x)]^{r}[1-F(x)]^{n-r} d x\right\} .
\end{aligned}
$$

$(B .2) \quad \mu_{r: n}^{(k)}=\alpha C_{r: n}\left\{I_{1}-I_{2}\right\}$

where,

$I_{1}=\int_{0}^{\infty} x^{k}(\lambda+x)^{-1}[F(x)]^{r-1}[1-F(x)]^{n-r} d x$.

By using integration by parts, we obtain

$$
\begin{aligned}
I_{1}=-\int_{0}^{\infty}\left[\left\{\left(\sum_{i=0}^{k-1}\left(\begin{array}{l}
k \\
i
\end{array}\right)(-1)^{i} \frac{1}{k-i}\left((\lambda+x)^{k-i}\right)\right)\right.\right. \\
+\log (\lambda+x)\}\left\{(r-1)[F(x)]^{r-2}[1-F(x)]^{n-r} f(x)\right. \\
\left.\left.-(n-r)[F(x)]^{r-1}[1-F(x)]^{n-r-1} f(x)\right\}\right] d x .
\end{aligned}
$$


Simplifying the above equation, we get

$$
\begin{aligned}
I_{1}=\sum_{i=0}^{k-1} \sum_{j=0}^{k-i}\left(\begin{array}{c}
k \\
i
\end{array}\right)\left(\begin{array}{c}
k-i \\
j
\end{array}\right)(-1)^{i} \lambda^{k-i-j}\left\{(n-r) \int_{0}^{\infty} x^{j}[F(x)]^{r-1}[1-F(x)]^{n-r-1} f(x) d x\right. \\
\left.-\quad(r-1) \int_{0}^{\infty} x^{j}[F(x)]^{r-2}[1-F(x)]^{n-r} f(x) d x\right\}+A_{1}-A_{2} \\
+\frac{r-1}{\alpha} \sum_{l=0}^{r-2}\left(\begin{array}{c}
r-2 \\
l
\end{array}\right)(-1)^{l} \frac{1}{(n+l-r+1)^{2}} \\
-\frac{n-r}{\alpha} \sum_{s=0}^{r-1}\left(\begin{array}{c}
r-1 \\
s
\end{array}\right)(-1)^{s} \frac{1}{(n+s-r)^{2}}
\end{aligned}
$$

Similarly,

$$
\begin{aligned}
& I_{2}=\sum_{i=0}^{k-1} \sum_{j=0}^{k-i}\left(\begin{array}{c}
k-1 \\
i
\end{array}\right)\left(\begin{array}{c}
k-i \\
j
\end{array}\right)(-1)^{i} \lambda^{k-i-j}\{(n \\
&-r) \int_{0}^{\infty} x^{j}[F(x)]^{r}[1-F(x)]^{n-r-1} f(x) d x \\
&\left.-\quad r \int_{0}^{\infty} x^{j}[F(x)]^{r-1}[1-F(x)]^{n-r} f(x) d x\right\}+A_{3}-A_{4} \\
&+\frac{r}{\alpha} \sum_{l=0}^{r-1}\left(\begin{array}{c}
r-1 \\
l
\end{array}\right)(-1)^{l} \frac{1}{(n+l-r+1)^{2}} \\
&-\frac{n-r}{\alpha} \sum_{s=0}^{r-1}\left(\begin{array}{c}
r-1 \\
s
\end{array}\right)(-1)^{s} \frac{1}{(n+s-r)^{2}}
\end{aligned}
$$

Substituting $I_{1}$ and $I_{2}$ into (B.2), we get the desired result.

\section{Proof of Theorem 3.3:}

$$
\begin{gathered}
\mu_{r ; s: n}^{\left(k_{1}, k_{2}\right)}=C_{r: s ; n} \int_{0}^{\infty} \int_{x}^{\infty} x^{k_{1}} y^{k_{2}}[F(x)]^{r-1}[F(y)-F(x)]^{s-r-1}[1 \\
-F(y)]^{n-s} f(x) f(y) d y d x
\end{gathered}
$$

or

$$
\text { = } C_{r: s ; n} \sum_{i=0}^{\substack{\mu_{r ; s: n}^{\left(k_{1}, k_{2}\right)} \\
n-s-r-1}} \sum_{j=0}^{s-r-s}\left(\begin{array}{c}
n-s \\
i
\end{array}\right)\left(\begin{array}{c}
s-r-1 \\
j
\end{array}\right)(-1)^{i+j} \int_{0}^{\infty} x^{k_{1}}[F(x)]^{j+r-1} f(x) I_{x} d x
$$

where,

$I_{X}=\int_{x}^{\infty} y^{k_{2}}[F(y)]^{s-r-j+i-1} f(y) d y$. 
Using (1.1) and (1.2), we have

(B. 4) $\quad I_{X}=\int_{x}^{\infty} y^{k_{2}}\left[1-\left(1+\frac{y}{\lambda}\right)^{-\alpha}\right]^{s-r-j+i-1} \frac{\alpha}{\lambda}\left(1+\frac{y}{\lambda}\right)^{-(\alpha+1)} d y$

Now simplifying the above expression, we get

$I_{X}=\frac{\alpha \lambda^{k_{2}} \sum_{l=0}^{k_{2}} \sum_{m=0}^{s-r-j+i-1}\left(\begin{array}{c}k_{2} \\ l\end{array}\right)\left(\begin{array}{c}s-r-j+i-1 \\ m\end{array}\right)(-1)^{l+m}}{l-k_{2}+\alpha m+\alpha}\left[\left(1+\frac{x}{\lambda}\right)^{-\alpha}\right]^{\frac{l-k_{2}}{\alpha}+m+1}$

Substituting $I_{X}$ in (B.3) and then simplifying it further, we get (3.3).

\section{Appendix C:}

\section{Proof of Theorem 4.1:}

Using (1.2) and the probability integral transformation $Y_{r}=F_{X}^{-1}\left(W_{r}\right)$, we have $F_{X}^{-1}\left(W_{r}\right)=\lambda\left[\left(1-W_{r}\right)^{\frac{-1}{\alpha}}-1\right]$.

Therefore,

$$
\text { (C.1) } \begin{aligned}
E_{g_{r}}\left[\log f_{X}\left(F_{X}^{-1}\left(W_{r}\right)\right)\right] & =E_{g_{r}}\left[\log f_{X}\left\{\lambda\left(\left(1-W_{r}\right)^{\frac{-1}{\alpha}}-1\right)\right\}\right] \\
& =\log \frac{\alpha}{\lambda}+\frac{\alpha+1}{\alpha}[\psi(n-r+1)-\psi(n+1)] .
\end{aligned}
$$

Substituting the value of (4.2) and (C.1) in (4.1), we get the desired result. 